\title{
Expanding the Use of the Ventrogluteal Region, an Evidence- Based Practice Example, in Senior Nursing Students: A Methadological Study
}

Meryem Kılıç, ( $\nabla$ meryemcal@gmail.com )

Sanko University

\section{Çisem Meteris}

Hacettepe University Hospital

Büşra Nur Kartal Biçer

Fırat Üniversitesi Hastanesi

\section{Research Article}

Keywords: Intramuscular, ventrogluteal region, injection, nursing student, evidence-based practice

Posted Date: October 11th, 2021

DOI: https://doi.org/10.21203/rs.3.rs-788133/v1

License: (c) (i) This work is licensed under a Creative Commons Attribution 4.0 International License. Read Full License 


\section{Abstract}

Intramuscular injections are administered into deep muscle tissue and can threaten human life if it is applied to the wrong area. Therefore, safe areas should be preferred for injection administration.

Purpose: The aim of this study was to evaluate the effect of the training on intramuscular injection to ventrogluteal region given to the senior nursing students on their knowledge, opinions and status of preferring this region .

Method: The study is a methodological study with single group and pretest- posttest design. The population of the study was composed of the senior nursing students $(\mathrm{N}=70)$ studying in SANKO University. 42 students completed the study. The data were collected with survey method.

Results :It was determined that the prejudices of the students about injection to ventrogluteal region changed significantly and positively after the training $(90.5 \%)$ and their information level increased $(p<0.05)$, but the students had negative thoughts about their self-confidence and about the possible objection of the patients $(p>0.05)$. Students scored the demonstration training as 8 out of $10(S D=2.03)$.

Conclusion: The study results indicated that the information level of the students about injection into the ventrogluteal region increased and their prejudices changed positively.

\section{Introduction}

The main condition of professionalisation in the nursing profession is undoubtedly to understand science and the scientific method adequately and reflect them onto the profession. Studies conducted using scientific method in nursing will move nurses from experience-based decisions to evidence-based decisions in the future. According to what is cited by Kocaman (2003), evidence-based practice started in 1972 with Dr. Archie Cochrane in the world. It was brought to agenda in Turkey in 2000 for the first time. Evidence-based practice is the selection of researches and results on a certain subject, synthesising the results, and preparing these results for decision of clinical practice (Kocaman, 2003). In order to conduct the nursing undergraduate education according to certain standards, Nursing National Core Education Program (NNCEP) was established in Turkey. Within the framework of NNCEP, it is mentioned that evidence-based practices should be taken into consideration in meeting the healthcare needs in nursing curriculum (NNCEP, 2014).

IMI (intramüscular) is a common treatment practice type applied frequently by nurses. For injection practice, selecting an area away from nerve, blood vessels and bone protrusions is an important issue for the practice (Kaya and Palloş, 2016; Lynn, 2015). In the literature, it is stated that dorsogluteal (DG) region, one of the IMI application areas, is a risky area for IMI application since there are rich blood vessels, subcutaneous tissue is thick compared to the other injection regions and it is close to sciatic nerve (Small, 2004; Nicoll and Hesby, 2002; Rodger and King, 2000; Lynn, 2015). Many studies have revealed that injections made into the DG region cause sciatic nerve injury (Kakati et al., 2013; Şener et al., 2014; Kadıoğlu, 2018). It has been stated in the studies that sciatic nerve injury develops due to injection practices made in DG region, and injection region and the technique used are the most important causes of sciatic nerve injury. Sciatic nerve injury causes paralysis, foot drop and deformities in some cases (Kakati et al., 2013; Şener et al., 2014; Kadıoğlu, 2018; Jung kim and Hyun Park, 2014; Small, 2004).

Evidence-based studies state that ventrogluteal (VG) region is the safest region since the thickness of subcutaneous tissue is lower than the other injection sites, it is far from the major nerves and blood vessels, the region is easily detected due to the possibility of determining bone protrusions, muscles in the region is large and developed (Small, 2004; Nicoll and Hesby, 2002; Rodger and King, 2000; Lynn, 2015).

Although VG region injection administrations are in the textbooks and shown in education (Lynn, 2015; Kaya and Palloş, 2016), it is seen that clinical nurses and nursing students mostly prefer DG region and less prefer VG region at high rates 
(Kaya et al. 2012; Tuğrul and Denat, 2014; Yavuz and Karabacak, 2011; Gülnar and Çalışkan, 2014; Güneş et al., 2009; Walsh and Brophy, 2011; Şanlıalp and Kuzu 2017; Alan and Çalışkan 2018; Šakić et al., 2012; Sarı et al., 2017; Arslan and Özden, 2018; Su and Bekmezci 2019). As the reason for this, nurses think that their knowledge level for VG region is not sufficient, they have never used this region, they do not know how to determine the region since the anatomic structure of the VG region is small, it is hard to position the patient while determining VG region, they are afraid to harm the patient, patients are not used to this region and the patients will not allow them to use the region. In addition, they stated that they do not prefer the VG region since they are used to other intramuscular injection sites, the patients will feel more pain if injection is made to the VG region, muscles in this region are not well developed, the region cannot be used in overweight and slim patients, they do not prefer VG region believing that the injector will touch the bone (Tuğrul and Denat, 2014; Gülnar and Çalışkan, 2014; Su and Bekmezci, 2020; Güneş et al., 2009; Korkmaz et al., 2018; Arslan and Özden, 2018; Eroğlu and Çevik, 2019).

Despite all these, the studies on increasing the use of VG region have revealed that the training given to the nurses increases their knowledge levels and the use of VG region (Arslan and Özden, 2018; Eroğlu and Çevik, 2019; Şanlıalp and Kuzu 2017; Öztürk et al., 2017).

In the light of all these data, it is seen that injection administration to VG region, an evidence-based practice example, is not preferred much and the trainings are effective in preference of VG region. We believe that it will be effective in realizing the evidence-based nursing practices repeated in nursing intern/last year's education, which is one step before the working life. Therefore, in this study, we planned to evaluate the effect of the training of injection to VG region to the students by repeating it.

\section{Hypotheses}

$H 1$ : The training positively affects the students' views about VG region.

$\mathrm{H} 2$ : The training increases the knowledge level of the students about VG region.

H3:The training increases the students' preference for VG region.

\section{Method}

\section{Type of the Study}

The study design is interventional with single group, pretest and posttest.

\section{Time and place of the study}

The study was conducted in the spring semester of 2019-202 academic year with the senior (intern) students of Nursing Department in the Faculty of Health Sciences of Gaziantep SANKO University. In our Nursing Department, theoretical and skill training about the injection administration to VG region are given to the students in the 2nd year course of Nursing Fundamentals and Practices. The course of Nursing Fundamentals and Practices is 17 hours (5-hour theoretical, 4-hour laboratory and 8-hour clinical practice). The skill practices are given in groups of 10 people in the basic skills laboratory. Injection basic skill practice to VG region is shown on a model of injection to VG region. Internship practice in our school is made in the spring term of the 4th year. Intern students do internships in departments of Internal Medicine Nursing, Surgical Diseases Nursing, Paediatric Nursing, Psychiatric Nursing, Public Health Nursing, and Obstetrics and Gynaecology Nursing. The learning outcomes of each clinical internship are determined by the departments and internship study programs are made in accordance with the learning outcomes in clinical internships. In the clinics, internship training is carried out with patient care, diagnostic and therapeutic interventions, and professional skills and practices. 


\section{Population and Sample}

The population of the study consisted of 70 intern students studying in the final year of nursing department of the Health Sciences Faculty in SANKO University. Sample selection was not used. It was planned to reach the entire population. However, since the research period coincided with the COVID-19 pandemic period, 42 students were able to complete the study.

\section{Data Collection Tools}

- Descriptive Characteristic Form: There are 5 questions (age, gender, graduated high school, order of preference, satisfaction with the choice of profession) including descriptive characteristics of the intern students in the first part.

- VG Region Opinion Form: The form was prepared by the researchers by benefiting from the literature (Tuğrul and Denat, 2014; Gülnar and Çalışkan, 2014; Su and Bekmezci, 2020; Güneş et al., 2009; Korkmaz et al., 2018; Arslan and Özden, 2018; Eroğlu and Çevik, 2019). In the form, there are 16 items evaluating the opinions about injecting the VG region. A five-point Likert type measurement system will be used in the evaluation of the items. These are "I strongly agree", "I agree", "Undecided”, "I disagree" and "I strongly disagree”. The assessment of the expressions about the opinions will be made as number and percentage.

- VG Region Information Form: The form was prepared by the researcher in line with the literature (Kaya and Palloş, 2016; Small, 2004; Nicoll and Hesby, 2002; Rodger and King, 2000 Süzen, 2015; Lynn, 2015). It was prepared to measure the knowledge levels about the injection administration to VG region. In the form, there are 20 suggestions about VG region determination, to whom it should be applied, preferring in slim and obese individuals, what kind of drugs to be used, whether it is painful, the properties of the subcutaneous tissue in the region and the safety of the region. In the assessment of the suggestions, true/false options are used. 11 of these questions were prepared as "True" and 9 were prepared as "False". The students' true responses to the suggestions get 1 point and wrong responses get 0 point. Knowledge score will be calculated over a total of 20 points. 10 points denote "moderate" success. The evaluation will be made as below $(<10)$ or above $(>10)$ average.

\section{Application Process of the Study}

1) At the beginning of the study, the students were informed about the purpose of the study and their consents for participating in the study were obtained. The pretest form was filled in the classroom and this process lasted for averagely 10-15 minutes. The forms were filled next to the researchers within averagely 10-15 minutes. While filling out the pretest form, there were 55 people in the classroom. Therefore, 55 people were included in the study. Theoretical training on injection administration in VG region was given to the students who were subjected to pretest. After the training, brochures on the subject were distributed to the students.

2) Since the students receive injection skill training in the second year and it is stated in the literature that problems are experienced in determining this region, determination of this region in particular was shown on a student who was not included in the study (Only the region was detected and displayed). Each student determined the area with his/her hand. The skill training made with demonstration method was provided in the clinics where the students did their internships. There were minimum 3 students and maximum 7 students in each training group. After the skill training, the students were given the opportunity to apply injection to VG region. However, due to the COVID-19 pandemic, the students were only able to go for practice for a week after VG region skill training. Since it was decided to conduct the education process of the students remotely in the world and in Turkey, the posttest of the study was applied to the students via an online survey 1 month after the training.

\section{Data Assessment}


The data were assessed using SPSS 24 package program. As descriptive statistics; mean and standard deviation or median and minimum-maximum values for continuous variables stated with measurement (age, VG region injection information score), frequency and percentage for qualitative variables (gender, graduated high school, order of preferring profession, satisfaction with profession, clinic where the internship was performed, injection practices, students' causes of not using VG region), and Wilcoxon test in the evaluation of opinions about VG region before and after the training were used.

\section{Ethics Committee}

For the study, approval was obtained from SANKO University Clinical Trials Ethics Committee on 27.02.2020 (Session No:2020/04, Decision No:02). Participation in the study was based on volunteerism. This study was conducted in accordance with the Principles of Helsinki Declaration.

\section{Results}

Table 1

Descriptive Characteristics of the Students ( $\mathrm{N}=42)$

\begin{tabular}{|c|c|c|}
\hline & \multicolumn{2}{|c|}{ Mean $\pm S D$ (min-max) } \\
\hline \multirow[t]{2}{*}{ Mean age (year) } & \multicolumn{2}{|c|}{$22.28 \pm 0.86(21-24)$} \\
\hline & $\mathbf{N}$ & $\%$ \\
\hline \multicolumn{3}{|l|}{ Gender } \\
\hline Male & 6 & 14.3 \\
\hline Female & 36 & 85.7 \\
\hline \multicolumn{3}{|l|}{ Graduated high school } \\
\hline Regular high school & 7 & 16.7 \\
\hline Anatolian and science high school & 30 & 71.4 \\
\hline Vocational high School & 4 & 9.5 \\
\hline Vocational school of health & 1 & 2.4 \\
\hline \multicolumn{3}{|c|}{ The order of choosing the nursing profession } \\
\hline 1st Preference & 27 & 64.3 \\
\hline 2nd Preference & 4 & 9.5 \\
\hline 3rd Preference & 4 & 9.5 \\
\hline 4th and higher & 7 & 16.7 \\
\hline \multicolumn{3}{|l|}{ Satisfaction with profession choice } \\
\hline Yes & 39 & 92.9 \\
\hline No & 3 & 7.1 \\
\hline \multicolumn{3}{|l|}{ The clinic where the internship is done } \\
\hline Internal medicine clinics & 22 & 52.38 \\
\hline Surgical clinics & 20 & 47.62 \\
\hline
\end{tabular}


Among 42 students participating in the study, 14.3\% (6) were male, 85.7\% (36) were female, the mean age was 22.28 (SD = 0.86 ( $\min =21, \max =24)$ and $71.4 \%(30)$ graduated from Anatolian and Science High School. 64.3\% (27) of the students preferred the nursing profession as their 1st preference and $92.9 \%$ (39) were satisfied with their choice of profession, $52.38 \%$ were doing their internship in internal medicine clinics, and $47.62 \%$ were doing their internship in surgical clinics (Table 1).

Table 2

Students' opinions and practice on IM injection before the training $(\mathrm{N}=42)$

\begin{tabular}{|c|c|c|}
\hline & $\mathbf{N}$ & $\%$ \\
\hline \multicolumn{3}{|l|}{ Have you administer an IMI? } \\
\hline Yes & 39 & 92.9 \\
\hline No & 3 & 7.1 \\
\hline \multicolumn{3}{|c|}{ Would you like the theoretical training for IMI to be repeated? } \\
\hline Yes & 27 & 65.3 \\
\hline No & 15 & 35.7 \\
\hline \multicolumn{3}{|l|}{ Would you like the skill training for IMI to be repeated? } \\
\hline Yes & 33 & 78.6 \\
\hline No & 9 & 21.4 \\
\hline \multicolumn{3}{|c|}{ Which one of the IMI regions did you use during your nursing education? } \\
\hline Ventrogluteal & 2 & 4.8 \\
\hline Dorsogluteal & 36 & 85.7 \\
\hline Deltoid & 11 & 26.2 \\
\hline Vastus lateralis & 2 & 4.8 \\
\hline Rectus femoris & 39 & 93.8 \\
\hline \multicolumn{3}{|l|}{ Reasons for not using VG region* } \\
\hline Not being used to this area & 7 & 16.7 \\
\hline Not having enough knowledge about this region & 4 & 9.5 \\
\hline Having fears because of not using this region before & 3 & 7.1 \\
\hline Preference of nurses in the clinic to use DG region & 11 & 26.2 \\
\hline
\end{tabular}

It was found that $92.9 \%$ (39) of the students made IMI during the training process. Of the students, $65.3 \%$ (27) wanted the theoretical training about IMI practices to be repeated and $78.6 \%$ wanted the skill training to be repeated. During the school education, students made injections mostly to the dorsogluteal (85.7\%) and rectus femoris (93.8\%) regions among IMI practices and only 2 students stated that they applied injection to VG region in this period. The reasons for students not to prefer VG region were as follows; the nurses did not use this region (26.2\%), they were not used to this area (16.7\%), they did not have sufficient knowledge about this region (9.5\%), and they had fears because they had never used this area (7.1\%), respectively (Table 2 ). 


\begin{tabular}{|c|c|c|}
\hline & $\mathbf{n}$ & $\%$ \\
\hline \multicolumn{3}{|l|}{ Status of making IMI } \\
\hline Ventrogluteal & 8 & 19.0 \\
\hline Dorsogluteal & 13 & 31.0 \\
\hline Those who made no injection** & 21 & 50.0 \\
\hline \multicolumn{3}{|l|}{ Reasons of the students, who preferred DG region, not for preferring VG region } \\
\hline The patient' request for the $D G$ region & 1 & 2.4 \\
\hline Being used to the DG area & 2 & 4.8 \\
\hline Thinking I haven't fully learned yet & 2 & 4.8 \\
\hline Nurses in the clinic prefer DG region & 5 & 12.0 \\
\hline \multicolumn{3}{|l|}{ How has the view about injecting the VG area changed? } \\
\hline Positively & 38 & 90.5 \\
\hline Negatively & 1 & 2.4 \\
\hline \multirow[t]{2}{*}{ Undecided } & 3 & 7.1 \\
\hline & \multicolumn{2}{|c|}{ Mean $\pm S D($ min-max $)$} \\
\hline The contribution of demonstration training on positive change of your thoughts about VG region & \multicolumn{2}{|c|}{$8 \pm 2.03(3-10)$} \\
\hline
\end{tabular}

Table 3 shows the effect of the training given to the students on their injection practices on VG region. After the training, $90.5 \%$ of the students evaluated that their views about injection to VG region changed positively and contribution of the training in the change of their opinion scored as 8 out of 10 points $(S D=2.03)$. After the training, it was determined that 9 students $(19.1 \%)$ made injections in VG region while $13(30.9 \%)$ of the students made injection to DG region and the remaining 21 (50\%) of the students had no opportunity to made injection due to pandemic period and absence of IM treatment in the clinic. As the reason for not applying injection to VG region, the students stated that the patients did not want this region [2.4\% (1)], nurses preferred the DG region [12\% (5)], they had not learnt the VG region fully [4.8\% (2)], and they were used to the DG region $[4.8 \%(2)]$.

Table 4

The students' mean scores from the information questions about VG region

\begin{tabular}{|llll|}
\hline & Mean \pm SD & Min - Max. & Z \\
& & & P \\
\hline Before the Training & $12.5 \pm 1.90$ & $8-16$ & $Z=-4.724$, \\
After the Training & $14.7 \pm 1.64$ & $11-18$ & $\mathrm{p}=0.000$ \\
\hline Wilcoxon test & & & \\
\hline
\end{tabular}

While knowledge level of the students about the injection to VG region before the training was $12.5 \pm 1.90$ ( $\min =8-\max =16)$, their knowledge level after the training increased significantly $[14.7 \pm 1.64(\min =11-\max =18)](Z=-4.724, p=0.000)(T a b l e$ 
Page $8 / 16$ 
Table 5

Students' views about applying injection to VG region (Before -After the Training)

\begin{tabular}{|c|c|c|c|c|c|c|c|c|c|c|c|}
\hline \multirow[t]{3}{*}{ Suggestions } & \multicolumn{2}{|c|}{ I strongly agree } & \multicolumn{2}{|l|}{ I agree } & \multicolumn{2}{|c|}{ Undecided } & \multicolumn{2}{|c|}{ I disagree } & \multicolumn{2}{|c|}{$\begin{array}{l}\text { I strongly } \\
\text { disagree }\end{array}$} & \multirow[t]{3}{*}{ Statistic } \\
\hline & BT & AT & BT & AT & BT & AT & BT & AT & BT & AT & \\
\hline & $\mathrm{n}(\%)$ & $\mathrm{n}(\%)$ & $\mathrm{n}(\%)$ & $\mathrm{n}(\%)$ & $\mathrm{n}(\%)$ & $\mathrm{n}(\%)$ & $\mathrm{n}(\%)$ & $\mathrm{n}(\%)$ & $n(\%)$ & $\mathrm{n}(\%)$ & \\
\hline \multirow{2}{*}{$\begin{array}{l}\text { 01: I think I } \\
\text { have enough } \\
\text { knowledge } \\
\text { about this } \\
\text { injection } \\
\text { administration }\end{array}$} & $\begin{array}{l}4 \\
(9.5)\end{array}$ & $\begin{array}{l}10 \\
(23.8)\end{array}$ & $\begin{array}{l}11 \\
(26.2)\end{array}$ & $\begin{array}{l}24 \\
(57.1)\end{array}$ & $\begin{array}{l}19 \\
(45.2)\end{array}$ & $\begin{array}{l}7 \\
(16.7)\end{array}$ & $\begin{array}{l}7 \\
(16.7)\end{array}$ & $\begin{array}{l}1 \\
(2.4)\end{array}$ & $\begin{array}{l}1 \\
(2.4)\end{array}$ & - & $\begin{array}{l}Z= \\
-3.953\end{array}$ \\
\hline & & & & & & & & & & & $\begin{array}{l}P= \\
0.000\end{array}$ \\
\hline \multirow{2}{*}{$\begin{array}{l}\text { O2: I have } \\
\text { concerns } \\
\text { about this } \\
\text { injection site. }\end{array}$} & $\begin{array}{l}4 \\
(9.5)\end{array}$ & - & $\begin{array}{l}14 \\
(33.3)\end{array}$ & $\begin{array}{l}3 \\
(7.1)\end{array}$ & $\begin{array}{l}19 \\
(45.2)\end{array}$ & $\begin{array}{l}13 \\
(31)\end{array}$ & $\begin{array}{l}5 \\
(11.9)\end{array}$ & $\begin{array}{l}21 \\
(50.0)\end{array}$ & - & $\begin{array}{l}5 \\
(11.9)\end{array}$ & $\begin{array}{l}Z= \\
-4.630\end{array}$ \\
\hline & & & & & & & & & & & $\begin{array}{l}P= \\
0.000\end{array}$ \\
\hline \multirow{2}{*}{$\begin{array}{l}\text { O3: I think } \\
\text { other injection } \\
\text { sites are more } \\
\text { useful. }\end{array}$} & $\begin{array}{l}5 \\
(11.9)\end{array}$ & $\begin{array}{l}4 \\
(9.5)\end{array}$ & $\begin{array}{l}14 \\
(33.3)\end{array}$ & $\begin{array}{l}13 \\
(31.0)\end{array}$ & $\begin{array}{l}17 \\
(40.5)\end{array}$ & $\begin{array}{l}9 \\
(21.4)\end{array}$ & $\begin{array}{l}6 \\
(14.3)\end{array}$ & $\begin{array}{l}12 \\
(28.6)\end{array}$ & - & $\begin{array}{l}4 \\
(9.5)\end{array}$ & $\begin{array}{l}Z= \\
-1.800\end{array}$ \\
\hline & & & & & & & & & & & $\begin{array}{l}P= \\
0.072\end{array}$ \\
\hline \multirow{2}{*}{$\begin{array}{l}\text { O4: I can } \\
\text { detect this } \\
\text { injection site } \\
\text { correctly. }\end{array}$} & $\begin{array}{l}7 \\
(16.7)\end{array}$ & $\begin{array}{l}10 \\
(23.8)\end{array}$ & $\begin{array}{l}10 \\
(23.8)\end{array}$ & $\begin{array}{l}23 \\
(54.8)\end{array}$ & $\begin{array}{l}19 \\
(45.2)\end{array}$ & $\begin{array}{l}7 \\
(16.7)\end{array}$ & $\begin{array}{l}5 \\
(11.9)\end{array}$ & $\begin{array}{l}2 \\
(4.8)\end{array}$ & $\begin{array}{l}1 \\
(2.4)\end{array}$ & - & $\begin{array}{l}Z= \\
-2.506\end{array}$ \\
\hline & & & & & & & & & & & $\begin{array}{l}P= \\
0.012\end{array}$ \\
\hline \multirow{2}{*}{$\begin{array}{l}\text { 05: I think this } \\
\text { injection site } \\
\text { is dangerous. }\end{array}$} & $\begin{array}{l}1 \\
(2.4)\end{array}$ & $\begin{array}{l}1 \\
(2.4)\end{array}$ & $\begin{array}{l}7 \\
(16.7)\end{array}$ & - & $\begin{array}{l}18 \\
(42.9)\end{array}$ & $\begin{array}{l}3 \\
(7.1)\end{array}$ & $\begin{array}{l}12 \\
(28.6)\end{array}$ & $\begin{array}{l}27 \\
(64.3)\end{array}$ & $\begin{array}{l}4 \\
(9.5)\end{array}$ & $\begin{array}{l}11 \\
(26.2)\end{array}$ & $\begin{array}{l}Z= \\
-3.922\end{array}$ \\
\hline & & & & & & & & & & & $\begin{array}{l}P= \\
0.000\end{array}$ \\
\hline \multirow{2}{*}{$\begin{array}{l}\text { 06: I think the } \\
\text { muscle size in } \\
\text { the area is } \\
\text { small. }\end{array}$} & $\begin{array}{l}2 \\
(4.8)\end{array}$ & $\begin{array}{l}2 \\
(4.8)\end{array}$ & $\begin{array}{l}10 \\
(23.8)\end{array}$ & $\begin{array}{l}11 \\
(26.2)\end{array}$ & $\begin{array}{l}20 \\
(47.6)\end{array}$ & $\begin{array}{l}7 \\
(16.7)\end{array}$ & $\begin{array}{l}9 \\
(21.4)\end{array}$ & $\begin{array}{l}14 \\
(33.3)\end{array}$ & $\begin{array}{l}1 \\
(2.4)\end{array}$ & $\begin{array}{l}8 \\
(19.0)\end{array}$ & $\begin{array}{l}Z= \\
-2.238\end{array}$ \\
\hline & & & & & & & & & & & $\begin{array}{l}P= \\
0.025\end{array}$ \\
\hline \multirow{2}{*}{$\begin{array}{l}\text { 07: I think it is } \\
\text { hard to } \\
\text { position the } \\
\text { patient. }\end{array}$} & $\begin{array}{l}5 \\
(11.9)\end{array}$ & $\begin{array}{l}1 \\
(2.4)\end{array}$ & $\begin{array}{l}8 \\
(19.0)\end{array}$ & $\begin{array}{l}4 \\
(9.5)\end{array}$ & $\begin{array}{l}12 \\
(28.6)\end{array}$ & $\begin{array}{l}3 \\
(7.1)\end{array}$ & $\begin{array}{l}14 \\
(33.3)\end{array}$ & $\begin{array}{l}20 \\
(47.6)\end{array}$ & $\begin{array}{l}3 \\
(7.1)\end{array}$ & $\begin{array}{l}14 \\
(33.3)\end{array}$ & $\begin{array}{l}Z= \\
-3.521\end{array}$ \\
\hline & & & & & & & & & & & $\begin{array}{l}P= \\
0.000\end{array}$ \\
\hline \multirow{2}{*}{$\begin{array}{l}\text { O8: I am } \\
\text { confident } \\
\text { about } \\
\text { applying } \\
\text { injection to } \\
\text { this region. }\end{array}$} & $\begin{array}{l}7 \\
(16.7)\end{array}$ & $\begin{array}{l}9 \\
(21.4)\end{array}$ & $\begin{array}{l}15 \\
(35.7)\end{array}$ & $\begin{array}{l}21 \\
(50.0)\end{array}$ & $\begin{array}{l}16 \\
(38.1)\end{array}$ & $\begin{array}{l}9 \\
(21.4)\end{array}$ & $\begin{array}{l}2 \\
(4.8)\end{array}$ & $\begin{array}{l}2 \\
(4.8)\end{array}$ & $\begin{array}{l}2 \\
(4.8)\end{array}$ & $\begin{array}{l}1 \\
(2.4)\end{array}$ & $\begin{array}{l}Z= \\
-1.635\end{array}$ \\
\hline & & & & & & & & & & & $\begin{array}{l}P= \\
0.102\end{array}$ \\
\hline \multirow{2}{*}{$\begin{array}{l}\text { 09: I think the } \\
\text { patient might } \\
\text { object to this } \\
\text { injection site. }\end{array}$} & $\begin{array}{l}8 \\
(19.0)\end{array}$ & $\begin{array}{l}6 \\
(14.3)\end{array}$ & $\begin{array}{l}13 \\
(31.0)\end{array}$ & $\begin{array}{l}18 \\
(42.9)\end{array}$ & $\begin{array}{l}13 \\
(31.0)\end{array}$ & $\begin{array}{l}13 \\
(31.0)\end{array}$ & $\begin{array}{l}7 \\
(16.7)\end{array}$ & $\begin{array}{l}5 \\
(11.9)\end{array}$ & $\begin{array}{l}1 \\
(2.4)\end{array}$ & - & $\begin{array}{l}Z= \\
-0.619\end{array}$ \\
\hline & & & & & & & & & & & $\begin{array}{l}P= \\
0.536\end{array}$ \\
\hline
\end{tabular}




\begin{tabular}{|c|c|c|c|c|c|c|c|c|c|c|c|}
\hline \multirow[t]{3}{*}{ Suggestions } & \multicolumn{2}{|c|}{ I strongly agree } & \multicolumn{2}{|l|}{ I agree } & \multicolumn{2}{|c|}{ Undecided } & \multicolumn{2}{|c|}{ I disagree } & \multicolumn{2}{|c|}{$\begin{array}{l}\text { I strongly } \\
\text { disagree }\end{array}$} & \multirow[t]{3}{*}{ Statistic } \\
\hline & BT & AT & BT & AT & BT & AT & BT & AT & BT & AT & \\
\hline & $\mathrm{n}(\%)$ & $\mathrm{n}(\%)$ & $\mathrm{n}(\%)$ & $\mathrm{n}(\%)$ & $\mathrm{n}(\%)$ & $\mathrm{n}(\%)$ & $\mathrm{n}(\%)$ & $\mathrm{n}(\%)$ & $\mathrm{n}(\%)$ & $\mathrm{n}(\%)$ & \\
\hline \multirow{2}{*}{$\begin{array}{l}\text { O10: I think } \\
\text { my skill level } \\
\text { is sufficient } \\
\text { for injection } \\
\text { administration } \\
\text { to this area. }\end{array}$} & $\begin{array}{l}6 \\
(14.3)\end{array}$ & $\begin{array}{l}6 \\
(14.3)\end{array}$ & $\begin{array}{l}5 \\
(11.9)\end{array}$ & $\begin{array}{l}18 \\
(42.9)\end{array}$ & $\begin{array}{l}14 \\
(33.3)\end{array}$ & $\begin{array}{l}10 \\
(23.8)\end{array}$ & $\begin{array}{l}11 \\
(26.2)\end{array}$ & $\begin{array}{l}8 \\
(19.0)\end{array}$ & $\begin{array}{l}6 \\
(14.3)\end{array}$ & - & $\begin{array}{l}Z= \\
-3.214\end{array}$ \\
\hline & & & & & & & & & & & $\begin{array}{l}P= \\
0.001\end{array}$ \\
\hline \multirow{2}{*}{$\begin{array}{l}011: \text { I think } \\
\text { the injector tip } \\
\text { will touch to } \\
\text { the bone } \\
\text { tissue during } \\
\text { the injection. }\end{array}$} & $\begin{array}{l}3 \\
(7.1)\end{array}$ & - & $\begin{array}{l}12 \\
(28.6)\end{array}$ & $\begin{array}{l}1 \\
(2.4)\end{array}$ & $\begin{array}{l}12 \\
(28.6)\end{array}$ & $\begin{array}{l}11 \\
(26.2)\end{array}$ & $\begin{array}{l}13 \\
(31.0)\end{array}$ & $\begin{array}{l}25 \\
(59.5)\end{array}$ & $\begin{array}{l}2 \\
(4.8)\end{array}$ & $\begin{array}{l}5 \\
(11.9)\end{array}$ & $\begin{array}{l}Z= \\
-3.833\end{array}$ \\
\hline & & & & & & & & & & & $\begin{array}{l}P= \\
0.000\end{array}$ \\
\hline \multirow{2}{*}{$\begin{array}{l}\text { 012: I do not } \\
\text { know how to } \\
\text { detect this } \\
\text { injection site. }\end{array}$} & $\begin{array}{l}3 \\
(7.1)\end{array}$ & $\begin{array}{l}3 \\
(7.1)\end{array}$ & $\begin{array}{l}13 \\
(31.0)\end{array}$ & $\begin{array}{l}4 \\
(9.5)\end{array}$ & $\begin{array}{l}9 \\
(21.4)\end{array}$ & $\begin{array}{l}5 \\
(11.9)\end{array}$ & $\begin{array}{l}13 \\
(31.0)\end{array}$ & $\begin{array}{l}20 \\
(47.6)\end{array}$ & $\begin{array}{l}4 \\
(9.5)\end{array}$ & $\begin{array}{l}10 \\
(23.8)\end{array}$ & $\begin{array}{l}Z= \\
-5.571\end{array}$ \\
\hline & & & & & & & & & & & $\begin{array}{l}P= \\
0.000\end{array}$ \\
\hline \multirow{2}{*}{$\begin{array}{l}\text { 013: I think it } \\
\text { is an area that } \\
\text { patients do } \\
\text { not encounter } \\
\text { in injection } \\
\text { practice. }\end{array}$} & $\begin{array}{l}10 \\
(23.8)\end{array}$ & $\begin{array}{l}11 \\
(26.2)\end{array}$ & $\begin{array}{l}16 \\
(38.1)\end{array}$ & $\begin{array}{l}25 \\
(59.5)\end{array}$ & $\begin{array}{l}9 \\
(21.4)\end{array}$ & $\begin{array}{l}4 \\
(9.5)\end{array}$ & $\begin{array}{l}5 \\
(11.9)\end{array}$ & $\begin{array}{l}1 \\
(2.4)\end{array}$ & $\begin{array}{l}2 \\
(4.8)\end{array}$ & $\begin{array}{l}1 \\
(2.4)\end{array}$ & $\begin{array}{l}Z= \\
-1.885\end{array}$ \\
\hline & & & & & & & & & & & $\begin{array}{l}P= \\
0.059\end{array}$ \\
\hline \multirow{2}{*}{$\begin{array}{l}\text { 014: I can } \\
\text { persuade } \\
\text { patients about } \\
\text { the injection } \\
\text { to this area. }\end{array}$} & $\begin{array}{l}3 \\
(7.1)\end{array}$ & $\begin{array}{l}8 \\
(19.0)\end{array}$ & $\begin{array}{l}19 \\
(45.2)\end{array}$ & $\begin{array}{l}22 \\
(52.4)\end{array}$ & $\begin{array}{l}17 \\
(40.5)\end{array}$ & $\begin{array}{l}10 \\
(23.8)\end{array}$ & $\begin{array}{l}3 \\
(7.1)\end{array}$ & $\begin{array}{l}2 \\
(4.8)\end{array}$ & - & - & $\begin{array}{l}Z= \\
-2.235\end{array}$ \\
\hline & & & & & & & & & & & $\begin{array}{l}P= \\
0.025\end{array}$ \\
\hline \multirow{2}{*}{$\begin{array}{l}\text { 015: I think } \\
\text { this injection } \\
\text { site may be } \\
\text { more painful. }\end{array}$} & $\begin{array}{l}3 \\
(7.1)\end{array}$ & - & $\begin{array}{l}7 \\
(16.7)\end{array}$ & - & $\begin{array}{l}26 \\
(61.9)\end{array}$ & $\begin{array}{l}4 \\
(9.5)\end{array}$ & $\begin{array}{l}5 \\
(11.9)\end{array}$ & $\begin{array}{l}24 \\
(57.1)\end{array}$ & $\begin{array}{l}1 \\
(2.4)\end{array}$ & $\begin{array}{l}14 \\
(33.3)\end{array}$ & $\begin{array}{l}Z= \\
-5.187\end{array}$ \\
\hline & & & & & & & & & & & $\begin{array}{l}P= \\
0.000\end{array}$ \\
\hline \multirow{2}{*}{$\begin{array}{l}\text { 016: I think } \\
\text { this injection } \\
\text { site will be } \\
\text { suitable for } \\
\text { slim patients. . }\end{array}$} & $\begin{array}{l}3 \\
(7.1)\end{array}$ & $\begin{array}{l}4 \\
(9.5)\end{array}$ & $\begin{array}{l}8 \\
(19.0)\end{array}$ & $\begin{array}{l}23 \\
(54.8)\end{array}$ & $\begin{array}{l}21 \\
(50)\end{array}$ & $\begin{array}{l}9 \\
(21.4)\end{array}$ & $\begin{array}{l}6 \\
(14.3)\end{array}$ & $\begin{array}{l}4 \\
(9.5)\end{array}$ & $\begin{array}{l}4 \\
(9.5)\end{array}$ & $\begin{array}{l}2 \\
(4.8)\end{array}$ & $\begin{array}{l}Z= \\
-2.616\end{array}$ \\
\hline & & & & & & & & & & & $\begin{array}{l}P= \\
0.009\end{array}$ \\
\hline
\end{tabular}

BT: Before the training AT: After the training

Table 5 shows the students' views about VG region before and after the training. It was seen that some negative thoughts of the students that prevented them to prefer $V G$ region $(01,02,04,05,06,07,010,011,012,014,015,016)$ after the training changed positively in a statistically significant way $(p<0.05)$. However, despite the training given, students still considered that the patients may object especially this region $(p=0.536)$ and their self-confidence was still insufficient $(p=$ 0.102).

\section{Discussion}

Interest, desire, and talent are at the forefront in choosing the nursing profession. Although nursing requires self-sacrifice, it is a profession that can be done with love (Özdelikara, Ağaçdiken and Aydın, 2016; Bölükbaş, 2018; Özveren, Gülnar and 
Özden, 2017; Kırağ, 2015). The results obtained from the study showed that $64.2 \%$ of the students preferred the nursing profession in their first choice and a great majority of the students (92.9\%) were satisfied with the nursing profession (Table 1). Nursing education is an education that has clinical practice. After learning basic nursing skills on models in a laboratory environment, students are expected to realize these skills in clinical environment (Bahar, 2015; Mete and Uysal, 2010; Sarmasoğlu, Dinç, Elçin, 2016). In the present study, it was seen that the majority of the students (92.9\%) made IMI during nursing education (Table 2). It was determined that among IMI administrations, students used DG region (95.7\%) and Rectus femoris region (93.8\%) at the highest rate and only 4.8\% (2) of the students made injection to VG region (Table 2). In their study, Karaahmetoğlu (2019) observed that the number of students preferring DG region was less (70.5\%) and those who preferred the VG region were more (55.5\%) compared to the present study. We think that this result is promising for the widespread use of injection administration to VG region.

VG region is far from large nerve and blood vessels and its subcutaneous tissue is thin. Less pain is felt in the injections made to this region and its location can be determined easily since it can be found by palpation. For all these reasons, it is stated in the literature that VG region can be used instead of DG region (Small, 2004; Nicoll and Hesby, 2002; Rodger and King, 2000). However, in other studies conducted with nursing students and clinical nurses in the literature, it is seen that DG region is mostly preferred in IMI, VG region is less preferred and knowledge about this region is insufficient (Kaya et al. 2012; Tuğrul and Denat, 2014; Yavuz and Karabacak, 2011; Gülnar and Çalışkan, 2014; Güneş et al., 2009; Walsh and Brophy, 2011; Şanlıalp and Kuzu 2017; Alan and Çalışkan 2018; Šakić et al. 2012; Sarı et al. 2017; Arslan and Özden, 2018; Su and Bekmezci 2019). In the present study, we believe that the knowledge levels of the nursing students about injection to VG region before the training were higher than the average $(12.5 \pm 1.90)$ but insufficient. It was determined that this score increased significantly 1 month after the training $(14.7 \pm 1.64)(p<0.05)$. We think that the information about drug administrations, which is one of the main duties of nurses, should be repeated during the internship period/senior year.

A similar study was conducted by Gülnar and Özveren (2016) with a group of working nurses. It was also seen in this study that the knowledge levels of nurses about VG region injection were higher than the average (13.53 \pm 2.50$)$ (range 0-24), but their knowledge levels increased significantly 4 months after the training (17.27 \pm 1.83$)$. Arslan and Özden (2018) made a study with single group and pretest-posttest design with 50 clinical nurses in order to increase their VG region preferences in IMI. In this study, it was determined that the nurses' knowledge levels about VG region was insufficient at the beginning (12.40 \pm 6.89$)$, but their knowledge level significantly increased after the training $(21.80 \pm 1.95)(P=0.05)$. Again, likewise in another quasi-experimental study conducted with 169 nurses, knowledge levels of the nurses before the training (10.4 \pm 2.17$)$ significantly increased after the training $(14.7 \pm 1.48)(p=0.001)$ (Şanlıalp and Kuzu 2017). It was seen in the present study that the knowledge levels of the students increased after the training but they were not as high as in these three studies (Table 4). We believe that this result was caused by the fact that the present study was conducted with a group of students who were not working in the field.

In the present study, the students expressed that their views about VG region positively changed after the training (90.5\%) and they would prefer VG region injection in the subsequent process. It was determined that the preference of the students for the VG region increased $(\% 19.1, n=8)$ compared to the period before the training but it was seen in the results that injection to DG region was still continuing $(30.9 \%, n=13)$ (Table 3). In the study conducted by Gülnar and Özveren (2018) $(n=81)$ with pretest-posttest experimental design, training about injection to VG region was given to the nurses and its effectiveness was evaluated. In their study, while $76.5 \%$ of nurses preferred DG region before the training, this rate decreased to $48.1 \%$ four months after the training. The number of nurses preferring VG region before the training increased from $7.4-34.6 \%$. The fact that nurses still preferred DG region although there was an increase in the nurses' preferences on VG region is similar to the present study results (Table 3). It is also seen in other studies that nurses' knowledge levels about VG region and frequency of preferring this region increased with the training (Sarı et al. 2017; Öztürk et al. 2017). It was shown that the most important reason affecting the students' preference on VG region before and after the training was the fact that clinical nurses did not prefer VG region (Table 3). Nursing students who do clinical practice do their treatment practices along with clinical nurses. We see that how nurses working in the clinic are important for students to put their 
knowledge learnt from the courses into the clinic, no matter how much evidence-based practices are emphasized in the course, being unaware of this practice and not using this practice for clinical nurses is more decisive in transferring these practices to the clinic. It is extremely important for clinical nurses to be aware of evidence-based practices in the literature and to put them into practice.

Due to the pandemic period, students could not do their internship 1 week after the training. Therefore, $21 \%$ of the students did not have the opportunity to do an injection (Table 3). The fact that the students could not participate in clinical practice due to the COVID 19 pandemic created a limitation in reaching our study goals. Despite this, $90.5 \%$ (38) of the students stated that their views about the VG region changed positively after the training. It was seen that they scored the contribution of the training given with demonstration on the positive change of their views as 8 out of 10 (SD=2.03) points.

In the studies, the reasons for nurses not to prefer VG region were shown mostly as the lack of knowledge about VG region, not being used to this region and inability to find this region. In addition, the nurses stated that they avoided the process since the patients may object to this area and may feel more pain and the injector may contact with the bone (Tuğrul and Denat, 2014; Gülnar and Çalışkan, 2014; Su and Bekmezci, 2020; Güneş et al., 2009; Korkmaz et al., 2018; Çevik and Eroğlu, 2019; Sarı et al. 2017; Arslan and Özden, 2018). Although training on IMI to VG region was provided to our students in the second year of nursing education, their opinions about VG region in the pretest are unfortunately similar to the other studies (Table 4). It was determined that the opinions of the students about the VG region increased significantly after the theoretical and skill training $(\mathrm{p}<0.05)$ (Table 4). Accordingly, it was found that the students' information levels about VG region, their self-confidences, their beliefs that they could give the correct position to the patient and convince them increased, and their concerns and fears about the region such as pain, correct determination of the region and applying it to slim people decreased. We think that the information repeated in the internship education, which is the last step before the students start working life, would have an effect on the realization of their knowledge. In the present study, before the training, $65.3 \%$ of our students requested the repetition of the theoretical training on IMI and $78.6 \%$ requested the repetition of the skill training. For this reason, we believe that the knowledge and practices about nursing skills applied the most by the students especially during internship period should be reviewed and supported with evidence-based practices. While doing so, it would also be appropriate to provide training about accessing evidence-based resources.

\section{Abbreviations}

NNCEP: Nursing National Core Education Program.

IMI: Intramuscular

DG: Dorsogluteal

VG: Ventrogluteal

\section{Declarations}

\section{Acknowledgements}

The authors would like to thank the nurse student and members of support groups who provided relevant information regarding the study. The authors also offer sincere appreciation to the health promoters for their cooperation during data collection.

\section{Author information}




\section{Affiliations}

Nursing Fundamentals Department of Nursing Fundamentals Dr. Instructor Member of Türkiye, Gaziantep , Meryem Kılıç.

Hacettepe University Hospital, Nurse, Türkiye, Ankara, Çisem Meteris.

Fırat University Hospital, Nurse, Türkiye, Elazığ, Büşra Nur Kartal Biçer.

\section{Corresponding author}

Meryem Kılıç.

\section{Contributions}

First author, second author, third author conceived the idea for the study. First author, second author, third author participated in the design of the study. First author was responsible for the statistical analysis plan and the sample size calculations. First author, participated in the desing of the study and in the analysis and interpretation of data, performed the statistical analysis and drafted the manuscript. First author, second author, third second were also involved in compiling patient information and data collection packs. All the authors have read and approved the final manuscript.

\section{Conpeting İnterests}

The authors declare that they have no competing interests.

\section{Ethics declarations}

\section{Ethics approval and consent to participate}

\section{Ethics Committee}

For the study, approval was obtained from SANKO University Clinical Trials Ethics Committee on 27.02.2020 (Session No:2020/04, Decision No:02). Participation in the study was based on volunteerism. This study was conducted in accordance with the Principles of Helsinki Declaration. All participants were informed about the study process and its objectives, and the written informed consent was obtained from them. The permission to the interviews was also obtained, and participants were assured about confidentiality and anonymity of the collected data. Participants had the right to withdraw from the study at any stage. However, no participants refused to participate in the study or dropped out of the study after enrollment.

\section{Consent for publication}

All participants were informed about the study process and its objectives, and the written informed consent was obtained from them.

\section{Competing interests}

The authors declare that they have no competing interests. 


\section{Funding}

Not applicable.

\section{Availability of data and materials}

The datasets generated and/or analysed during the current study are not publicly available due [restrictions by the Research and Ethics Committee in the Faculty of Nursing at Sanko University to protect the participants' privacy] but are available from the corresponding author on reasonable request.

\section{References}

1. Alan, S., Çalışkan, N., 2018. Knowledge Level About Intramuscular Injectıon Application of Nursing Last Grade Students. Ege University Nursing Faculty Journal, 34 (1), 36-53 (in Turkish): 36787/340142.

2. Arslan, G. G., Özden, D., 2018. Creating a change in the use of ventrogluteal site for intramuscular injection. Patient preference and adherence, 12, 1749, DOI: 10.2147/PPA.S168885.

3. Bahar, A., 2015. An Innovation in Basic Nursing Skills Education Web Based. Anatolian Journal of Nursing and Health Sciences, 18(4), 304-311 (in Turkish), DOI: 10.17049/ahsbd.24118.

4. Bölükbaş, N., 2018. Nursing students' choice of profession and influencing factors. Ordu University Journal of Nursing Studies, 1 (1), 10-17 (in Turkish): 41738/456765.

5. Eroğlu, S., Çevik, K., 2019. The Effect of Neuro Linguistic Programmıng (NLP) Techınıques On Nurses And Mıdwıves On Intramuscular Injection Application In The Ventrogluteal Area.Acıbadem Üniversitesi Sağlık Bilimleri Dergisi, 10 (4) (in Turkish), DOI : 10.31067/0.2019.125.

6. Gülnar, E., Çalışkan, N., 2014. Determination of knowledge level of nurses about intramuscular injection application to ventrogluteal region. Dokuz Eylul University Faculty of Nursing Electronic Journal, 7 (2), $70-77$ (in Turkish): $46808 / 586997$.

7. Gülnar, E., Özveren, H., 2016. An evaluation of the effectiveness of a planned training program for nurses on administering intramuscular injections into the ventrogluteal site. Nurse education today, 36, 360-363, DOI: 10.1016/j.nedt.2015.09.001.

8. Güneş, Ü. Y., Zaybak, A., Biçiçi, B., Çevik, K., 2009. Investıgation of Nurses Applications for Intramuscular Injection Process. Anatolian Journal of Nursing and Health Sciences, 12(4), 84-90 (in Turkish): 46808/586997.

9. HUÇEP, 2014. National Nursing Core Educatıon Program. Retrieved 07 September 2020. Available form: http://dijitalhemsire.net/wp-content/uploads/hucep-2014-pdf.pdf (in Turkish): 41130/497157.

10. Jung Kim, H., Hyun Park, S., 2014. Sciatic nerve injection injury. Journal of International Medical Research, 42(4), 887897, DOI: 10.1177 / 0300060514531924.

11. Kadıoğlu, H. H., 2018. Sciatic Nerve Injuries from Gluteal Intramuscular Injection According to Records of the High Health Council. Turkish neurosurgery, 28(3), 474-478, DOI: 10.5137 / 1019-5149.JTN.19789-16.4.

12. Kakati, A., Bhat, D., Devi, B. I., Shukla, D., 2013. Injection nerve palsy. Journal of neurosciences in rural practice, 4(1), 13, DOI: 10.4103/0976-3147.105603.

13. Karaahmetoğlu, G.U. Investigation of the Knowledge Level of Nursing Students About Intramuscular Injection. Abant Medical Journal, 8 (3), 155-161 (in Turkish), DOI: 10.5505/abantmedj.2019. 81894.

14. Kaya, N. ve Palloş, A., 2016 . Parenteral İlaç Uygulamaları. T. A. Aştı \& A. Karadağ (Ed.), Hemşirelik Esasları (s. 787-794). İstanbul: Akademi Basın ve Yayıncılık, Turkey. (in Turkish).

15. Kaya, N., Turan, N., Palloş, A.Ö., 2012. Shouldn't the dorsogluteal area be used for intramuscular injection? Florence Nihtingale Nursing Journal, 20 (2), 146-183 (in Turkish), DOI: 10.1016/j.ijnurstu.2014.07.002. 
16. Kırağ, N.,2015. Factors associated with choosing the nursing profession. Dokuz Eylul University Faculty of Nursing Electronic Journal, 8 (4), 226-231 (in Turkish). Retrieved from https://dergipark.org.tr/tr/pub/deuhfed/issue/46801/586843.

17. Kocaman, G., 2003. Evidence-Based Practice in Nursing Journal of Nursing Research Development, 5 (2), $61-69$ (in Turkish), DOI: 10.1111/jocn.13586.

18. Korkmaz, E., Karagözoğlu, Ş., Çerik, B. K., Yıldırım, G., 2018. Information Status and Application Preferences of Nurses About Intramuscular Injection Areas. Turkish Journal of Research \& Development in Nursing, 20 (1) (in Turkish).

19. Lynn, P., 2015. Taylor Klinik Hemşirelik Becerileri. (H. Bektaş, Çev.). Ankara: Nobel Akademik Yayıncılık Eğitim Danışmanlık. (Orijinal yayının basım yılı 2011). (s. 190-198).(in Turkish).

20. Mete, S., Uysal, N., 2010. Evaluation of Psychomotor Skills Training in Nursing Professional Skills Laboratory by Studenst and Trainers Turkish Journal of Research \& Development in Nursing, 12(2) (in Turkish). Retrieved from https://dergipark.org.tr/tr/pub/hemarge/issue/52709/695117.

21. Nicoll, L. H., Hesby, A., 2002. Intramuscular injection: an integrative research review and guideline for evidence-based practice. Applied nursing research, 15(3), 149-162, DOI: 10.1053/apnr.2002.34142.

22. Özdelikara, A., Ağaçdiken, S., Aydın, E., 2016. Hemşirelik Öğrencilerinin Meslek Seçimi ve Etkileyen Faktörler. ACU Sağlık Bil Derg 2016(2):83-88 (in Turkish). http://www.acibadem.dergisi.org/uploads/pdf/pdf_AUD_347.pdf.

23. Öztürk, D., Baykara, Z. G., Karadağ, A., Eyikara, E., 2017. The effect of in-service education on nurses' preference for the ventrogluteal site in intramuscular injection implementation. Journal of Human Sciences, 14(4), 4199-4205, DOI:10.14687/jhs.v14i4.

24. Özveren, H., Gülnar, E., Özden, D., 2017. Determination of factors affecting nursing students' choice of profession. Turkish Journal of Clinics and Laboratory, 8(2), 57-64 (in Turkish), DOI: 10.18663/tjcl.320040.

25. Rodger, M. A., King, L., 2000. Drawing up and administering intramuscular injections: a review of the literature. Journal of advanced nursing, 31(3), 574-582, DOI: 10.1046/j.1365-2648.2000.01312.x.

26. Šakić, B., Milutinović, D., Simin, D., 2012. An assessment of intramuscular injection practices among nursing students and nurses in hospital settings: is it evidence-based. South eastern Europe health sciences journal, 2(2), 114-21.

27. Şanlıalp, Z. A., Kuzu, K. N., 2017. The Effect of Training on Intramuscular Injection Knowledge and Practies of Nurses Z Technique and Ventrogluteal Area. Journal of Nursing Research Development, 19(1), 26-37 (in Turkish): 2011 SBE011.

28. Sarı, D., Şahin, M., Yaşar, E., Taşkiran, N., Telli, S., 2017. Investigation of Turkish nurses frequency and knowledge of administration of intramuscular injections to the ventrogluteal site: results from questionnaires. Nurse Education Today, 56, 47-51, DOI: 10.1016/j.nedt.2017.06.005.

29. Sarmasoğlu, Ş., Dinç, L., Elçin, M., 2016. Nursing students' views on standard patients and models used in clinical skills training. Journal of Nursing Education and Research, 13 (2), 107-115 (in Turkish), DOI: 10.5222/HEAD.2016.107.

30. Şener, M.T., Kır, M.Z., Şahingöz, S., Ancı., Kök, A. N., 2014. Sciatic Nerve İnjury Legal Cases. Düzce Medical Journal, 16(1): 48289/611393.

31. Small, S. P., 2004. Preventing sciatic nerve injury from intramuscular injections: literature review. Journal of advanced nursing, 47(3), 287-296, doi.org/10.1111/j.1365-2648.2004.03092.x.

32. Su, S., Bekmezci, E., 2020. The Reasons for Nurses not to Use Ventrogluteal Region in Intramusular Injection Administration Journal of Education and Research in Nursing, 17(1), 46-51, DOI: 10.5222/HEAD.2020.046.

33. Süzen, L. B., 2015. İlaç Uygulamaları. F.A. Ay (Ed.), Sağlık Uygulamalarında Temel Kavramlar ve Beceriler (s. 506-514) İstanbul: Nobel Tıp Kitabevleri, Turkey. (in Turkish).

34. Tuğrul, E., Denat, Y., 2014. Nurses' Knowledge, Opinion and Practices Regarding Ventrogluteal Area Injection. Dokuz Eylul University Faculty of Nursing Electronic Journal, 7 (4) (in Turkish), DOI: 10.2147 / PPA.S168885.

35. Walsh, L., Brophy, K., 2011. Staff nurses' sites of choice for administering intramuscular injections to adult patients in the acute care setting. Journal of advanced nursing,67(5), 1034-1040, DOI: 10.1111/j.1365-2648.2010.05527.x.

Page 15/16 
36. Yavuz, D. E., Karabacak, Ü., 2011. Why should we choose the ventrogluteal region for intramuscular injection? Nursing Research and Development Journal, 13 (2), 81-88 (in Turkish): 52714/695170. 